\title{
Erratum to: MicroRNAs involved in the browning process of adipocytes
}

\author{
N. Arias • L. Aguirre • A. Fernández-Quintela • \\ M. González • A. Lasa • J. Miranda • M. T. Macarulla • \\ M. P. Portillo
}

Published online: 16 March 2016

(C) University of Navarra 2016

\section{Erratum to: Journal of Physiology and Biochemistry DOI 10.1007/s13105-015-0459-z}

The original version of this article was corrected because this mini review unfortunately contained a mistake regarding the role of miR-26 in the regulation of white adipose tissue browning. The current version available on SpringerLink is already corrected. This erratum summarizes the corrections made for all readers who possibly downloaded the previous version:

In the abstract, the correct sentence is the one below: "These studies have shown, by using either primary adipocyte cultures or experimental models of mice (KO mice, mice overexpressing a specific miRNA), that

The online version of the original article can be found at http:// dx.doi.org/10.1007/s13105-015-0459-z.

N. Arias · L. Aguirre · A. Fernández-Quintela · A. Lasa · J. Miranda $\cdot$ M. T. Macarulla • M. P. Portillo

Nutrition and Obesity Group, Department of Nutrition and Food Sciences, University of Basque Country (UPV/EHU) and Lucio Lascaray Research Center, Vitoria-Gasteiz, Spain

N. Arias $\cdot$ L. Aguirre $\cdot$ A. Fernández-Quintela $\cdot$ A. Lasa $\cdot$ J. Miranda $\cdot$ M. T. Macarulla $\cdot$ M. P. Portillo $(\bowtie)$ CIBER Fisiopatología de la Obesidad y Nutrición (CIBERobn), Instituto de Salud Carlos III, Madrid, Spain

e-mail: mariapuy.portillo@ehu.eus

M. González

Nutrition and Food Science, Faculty of Biochemistry and Biological Sciences, National University of Litoral, Santa Fe, Argentina
miR-196a, miR-26, and miR-30 are needed for browning process development. By contrast, miR-155, miR133, miR-27b, and miR-34 act as negative regulators of this process."

Instead of the one below:

"These studies have shown, by using either primary adipocyte cultures or experimental models of mice (KO mice, mice overexpressing a specific miRNA) that miR-196a and miR-30 are needed for browning process development. By contrast, miR-155, miR-133, miR$27 \mathrm{~b}, \mathrm{miR}-34$ and miR-26 act as negative regulators of this process."

In miR-26 in the first and second paragraphs, the correct sentences are the following:

"In this study, they transfected hMADS cells with miR-26a, and they observed an upregulation of brown/brite adipocyte markers."

"They confirmed the downregulation of ADAM17 mRNA and protein by miR-26a and miR-26b, as well as the upregulation of ADAM17 after miR-26a/b inhibition. Finally, luciferase reporter assays confirmed a direct interaction of miR-26a with the ADAM17 3' UTR. Due to the fact that downregulation of ADAM17 was able to increase $U c p 1$ expression in hMADS cells, these authors suggested the involvement of ADAM17 in the promotion of brown adipocyte characteristics."

Instead of the following:

"In this study, they transfected hMADS cells with antisense oligonucleotides against miR-26a, 
inhibiting miR-26a and -26b. No differences were observed in lipid accumulation between both transfected and non-transfected cells. By contrast, in the case of gene expression, up-regulation of brown/brite adipocyte markers"

"They observed that the inhibition of miR-26a/b led to the up-regulation of ADAM17 gene and protein expressions, which in turn repressed Ucpl expression. Based on these results, the authors suggested that repressing miR-26a/b should decrease the browning process."
In the general conclusion, the correct sentence is the one below:

"Thus, miR-196a, miR-26, and miR-30 act as positive regulators and miR-155, miR-133, miR-27b, and miR34 as negative regulators."

Instead of the one below:

"Thus, miR-196a, and miR-30 act as positive regulators, and miR-155, miR-133, miR-27b, miR-34 and miR-26 as negative regulators." 